\title{
On the solution of a boundary value problem related to the heat transmission
}

\author{
Khanlar R. Mamedov, Volkan Ala \\ Mathematics Department, Science and Letters Faculty, Mersin University, Mersin, Turkey \\ Email address: \\ hanlar@mersin.edu.tr (K.R. Mamedov), volkanala@mersin.edu.tr (V. Ala)
}

To cite this article:

Khanlar R. Mamedov, Volkan Ala. On the Solution of a Boundary Value Problem related to the Heat Transmission. American Journal of Applied Mathematics. Vol. 2, No. 2, 2014, pp. 54-59. doi: 10.11648/j.ajam.20140202.12

\begin{abstract}
In this study, we consider a heat transmission problem which has derivative with respect to the time in boundary condition. Applying the seperation of variables method, we get a Sturm-Liouville equation with discontinuous coefficient and a spectral parameter dependent boundary condition. For this spectral problem, the operator theoretic formula is given, the resolvent operator constructed and the expansion formula with respect to the eigenfunctions obtained. Using the expansion formula, the solution of the heat problem expressed.
\end{abstract}

Keywords: Sturm-Liouville Operator, Resolvent Operator, Expansion Formula

\section{Introduction}

In this paper, we consider the following heat transmission problem:

$$
\begin{gathered}
\rho(x) \frac{\partial v}{\partial t}=\frac{\partial^{2} v}{\partial x^{2}}-q(x) v, \quad x>0, \quad t>0 \\
\left.\left(-\beta_{0} v+\frac{\partial v}{\partial x}+\beta_{1} \frac{\partial v}{\partial t}\right)\right|_{x=0}=0, \quad t>0, \\
v(x, 0)=\Phi_{0}(x), \quad x>0,
\end{gathered}
$$

where

$$
v(0,0)=\vartheta_{0}
$$

In keeping with the physics of the problem, we assume that the boundary condition at $x=\infty$, the solution $v(x, t)$ remains bounded:

$$
\lim _{x \rightarrow \infty}|v(x, t)|<\infty
$$

$q(x)$ is a real-valued function integrable on $[0,+\infty)$ satisfying the following condition:

$$
\int_{0}^{\infty} x|q(x)| d x<\infty
$$

$\beta_{0}, \beta_{1}<0$ are real numbers, $\rho(x)$ is the following piecewise-constant function which has discontinuity at point $a \in[0,+\infty)$.

$$
\rho(x)=\left\{\begin{array}{cc}
\alpha^{2}, & 0 \leq x<a, \\
1, & x \geq a .
\end{array}\right.
$$

with $0 \leq \alpha \neq 1$.

We assume that $\Phi_{0}(x)$ is sufficiently smooth continuous function which has first and second continuous derivatives for $x>0$. Suppose that, $\Phi_{0}{ }^{\prime \prime}(x)=O\left(x^{-2}\right)$ as $x \rightarrow \infty, x>0$. $\Phi_{0}(x)=O\left(x^{-1}\right)$ as $x \rightarrow \infty$.

Problem (1)-(4) was researched for the case of $\rho(x)=1, q(x)=0, \beta_{0}=0, \beta_{1}=1$ by Cohen [1].

A semi-infinite right cylindrical solid with cross section of arbitrary shape and size and with plane terminal face at $x=0$ has its lateral surface insulated against the heat conduction and has an initial temperature distribution depending on only the longitudinal coordinate $x$.

A liquid of arbitrary initial temperature is kept well stirred to insure at each instant a uniform temperature throughout it, at time $t=0$ the plane terminal face of the solid is placed in contact with the liquid. For simplicity it is assumed that the liquid is not accessible to a transfer of heat from its surrounding medium. The problem is to determine the temperature of the liquid and the distribution of temperature in the solid at any time $t>0$. 
When the seperation of variables method is used for problem (1),(2) a spectral problem is obtained which involves eigenvalue in boundary condition. Generally, with these type of boundary conditions are encountered when the boundary conditions of the main problem contain partial derivative with respect to the time.

Applying the method of seperation of variables to problem (1),(2) we get the spectral problem below:

$$
\begin{aligned}
& -y^{\prime \prime}+q(x) y=\lambda^{2} \rho(x) y, \\
& y^{\prime}(0)-\left(\beta_{0}+\beta_{1} \lambda^{2}\right) y(0)=0,
\end{aligned}
$$

where

$$
\lim _{x \rightarrow \infty}|y(x)|<\infty
$$

The spectral analysis of (5) was investigated for finite interval in [2] and for infinite interval in [3]. Expansion formula for Sturm-Liouville problem is given in [4-9] and etc. Direct scattering problem of $(5),(6)$ for the case $\beta_{0}=0$ and $\beta_{1}=1$ was examined in [10]. The inverse problem of scattering theory with discontinuous coefficient for (5) was studied in [12-14].

This paper is organised as follows: In Section 1, we give the integral representation for a second order differential equation with a discontinuous coefficient and the spectral properties of boundary value problem. In Section 2, the operator theoretic formulation of problem (5),(6) in the Hilbert space is defined. In Section 3, the resolvent operator is constructed. In Section 4, the expansion formula with respect to the eigenfunctions is obtained and finally in Section 5, we get formula for the solution of the heat transmission problem (1)-(4) using the expansion formula with respect to the eigenfunctions.

It is known from [14] that for all $\lambda$ from the closed upper half-plane, (5) has a unique Jost solution $f(x, \lambda)$ which can be represented in the form below:

$$
f(x, \lambda)=f_{0}(x, \lambda)+\int_{\mu^{+}(x)}^{\infty} K(x, t) e^{i \lambda t} d t
$$

where $\mu^{+}(x)=x \sqrt{\rho(x)}+a(1-\sqrt{\rho(x)})$ and the kernel $K(x, t)$ satisfies the inequality,

$$
\int_{\mu^{+}(x)}^{\infty}|K(x, t)| d t \leq c \int_{x}^{\infty} t|q(t)| d t, \quad 0<c=\text { const } .
$$

For real $\lambda \neq 0$, the functions $f(x, \lambda)$ and $\overline{f(x, \lambda)}$ form the fundamental system of solutions of equation (5) and the Wronskian of this system is equal to $2 i \lambda$ :

$W\{f(x, \lambda), \overline{f(x, \lambda)}\}=f^{\prime}(x, \lambda) \overline{f(x, \lambda)}-f(x, \lambda) \overline{f(x, \lambda)}=2 i \lambda$. Let be $\omega(x, \lambda)$ the solution of (5) satisfying the initial data

$$
\omega(0, \lambda)=1, \omega^{\prime}(0, \lambda)=\beta_{0}+\beta_{1} \lambda^{2}
$$

We define

$$
\varphi(\lambda):=f^{\prime}(0, \lambda)-\left(\beta_{0}+\beta_{1} \lambda^{2}\right) f(0, \lambda)
$$

Using the form (7) for the solution of (5),(6) it can be proved that $\varphi(\lambda)$ may have only a finite number of zeros in the half plane $\operatorname{Im} \lambda>0$. Moreover, all these zeros are simple and lie on the imaginary axis. These propositions are proved by the same method that was used in [7] (Lemma 3.1.6).

(Also see [11], Lemma 3 and [13], Lemma 2.)

Let us denote differentiation with respect to $\lambda$ with a dot and let $\lambda=i \lambda_{j}\left(\lambda_{j}>0\right)$ be the zeros of $\varphi(\lambda)$ in the half plane $\operatorname{Im} \lambda>0$. For $\lambda=i \lambda_{j}, j=1,2, \ldots, n$, we get

$$
\begin{aligned}
m_{j}^{-2} & =\int_{0}^{\infty} \rho(x)\left|f\left(x, i \lambda_{j}\right)\right|^{2} d x+\beta_{1}\left|f\left(0, i \lambda_{j}\right)\right|^{2} \\
& =-\frac{1}{2 i \lambda_{j}} \dot{\varphi}\left(i \lambda_{j}\right) f\left(0, i \lambda_{j}\right) .
\end{aligned}
$$

The numbers $m_{j}^{-2}$ are called the normalizing numbers of problem (5),(6). (see [13], formula (1.14))

\section{The Operator Theoretic Formulation}

In the Hilbert Space $H_{\rho}=L_{2, \rho}(0, \infty) \times \square$ an inner product is defined by

$$
\langle F, G\rangle:=\int_{0}^{\infty} F_{1}(x) \overline{G_{1}(x)} \rho(x) d x-\frac{1}{\beta_{1}} F_{2} \overline{G_{2}}
$$

where

$$
F=\left(\begin{array}{c}
F_{1}(x) \\
F_{2}
\end{array}\right), \quad G=\left(\begin{array}{c}
G_{1}(x) \\
G_{2}
\end{array}\right) \in H_{\rho}
$$

For convenience, we put

$$
\begin{gathered}
R_{0}[y]=\beta_{0} y(0)-y^{\prime}(0), \\
R_{0}^{\prime}[y]=\beta_{1} y(0) .
\end{gathered}
$$

Let us define operator (see [1].)

$$
L(F):=\left(\begin{array}{c}
l\left(F_{1}\right) \\
F_{1}^{\prime}(0)
\end{array}\right) \text {. }
$$

with domain

$$
D(L):=\left\{\begin{array}{c}
F \in H_{\rho} \mid F_{1}(x), F_{1}^{\prime}(x) \text { are } \mathrm{AC}[0, b] \subset[0, \infty), \\
l\left(F_{1}\right) \in L_{2, \rho}(0, \infty), F_{2}=\beta_{1} F_{1}(0) .
\end{array}\right\}
$$

(AC: absolutely continuous) where 


$$
l\left(F_{1}\right)=\frac{1}{\rho(x)}\left\{-F_{1}^{\prime \prime}+q(x) F_{1}\right\} .
$$

Problem (5),(6) is equivalent to the equation $L y=\lambda^{2} y$ and the operator $L$ with the domain $D(L)$ is self-adjoint in the Hilbert space of $H_{\rho}$.

\section{The Resolvent Operator}

We suppose that $\lambda^{2}$ is not a spectrum point of operator $L$, then the resolvent $R_{\lambda^{2}}=\left(L-\lambda^{2} I\right)^{-1}$ exists. Now, let us find this expression of the operator $R_{\lambda^{2}}(L)$.

Theorem 1: All numbers of the form $\lambda^{2}, \operatorname{Im} \lambda>0$ $\varphi(\lambda) \neq 0$ belong to the resolvent set of the operator $L$. The resolvent $R_{\lambda^{2}}$ is the integral operator with the kernel which has the following form:

$$
\begin{gathered}
R_{\lambda^{2}}(L)=\int_{0}^{\infty} G(x, \xi ; \lambda) F(\xi) d \xi, \\
G(x, \xi ; \lambda)=\frac{-1}{\varphi(\lambda)} \begin{cases}\omega(x, \lambda) f(\xi, \lambda), & x \leq \xi \leq \infty, \\
f(x, \lambda) \omega(\xi, \lambda), & 0 \leq \xi<x .\end{cases}
\end{gathered}
$$

Proof: Let $F \in D(L)$ and $F=\left(\begin{array}{c}F_{1}(x) \\ F_{2}\end{array}\right)$ be zero in exterior of every finite interval $[0, b] \subset[0, \infty)$. To construct the resolvent operator of $L$, we need to solve the initial value problem

$$
\begin{aligned}
-y^{\prime \prime}+q(x) y & =\lambda^{2} \rho(x) y+\rho(x) F_{1}(x), \\
y^{\prime}(0) & =\left(\beta_{0}+\beta_{1} \lambda^{2}\right) y(0)+F_{2} .
\end{aligned}
$$

By applying the variation of constants, we want to find the solution of problem $(11),(12)$ which has a form

$$
y(x, \lambda)=c_{1}(x, \lambda) \omega(x, \lambda)+c_{2}(x, \lambda) f(x, \lambda),
$$

where $\omega(x, \lambda)$ and $f(x, \lambda)$ are solutions of homogenous problem for $\operatorname{Im} \lambda>0$.

Hence, we get

$$
\left\{\begin{array}{c}
c_{1}^{\prime}(x, \lambda) \omega(x, \lambda)+c_{2}^{\prime}(x, \lambda) f(x, \lambda)=0, \\
c_{1}^{\prime}(x, \lambda) \omega^{\prime}(x, \lambda)+c_{2}^{\prime}(x, \lambda) f^{\prime}(x, \lambda)=-\rho(x) F_{1}(x) .
\end{array}\right.
$$

Since $y(x, \lambda) \in L_{2, \rho}(0, \infty)$, then $c_{1}(\infty, \lambda)=0$.

Using this relation and (14), we obtain

$$
\left\{\begin{array}{c}
c_{1}(x, \lambda)=-\int_{x}^{\infty} \frac{f(\xi, \lambda)}{\varphi(\lambda)} F_{1}(\xi) \rho(\xi) d \xi \\
c_{2}(x, \lambda)=c_{2}(0, \lambda)-\int_{0}^{x} \frac{\omega(\xi, \lambda)}{\varphi(\lambda)} F_{1}(\xi) \rho(\xi) d \xi
\end{array}\right.
$$

Substituting (15) into (13), we obtain

$$
y(x, \lambda)=\int_{0}^{\infty} G(x, \xi ; \lambda) F_{1}(\xi) \rho(\xi) d \xi+c_{2}(0, \lambda) f(x, \lambda) .
$$

Taking the condition (12), we find $c_{2}(0, \lambda)=\frac{F_{2}}{\varphi(\lambda)}$.

Thus,

$$
y(x, \lambda)=\int_{0}^{\infty} G(x, \xi ; \lambda) F_{1}(\xi) \rho(\xi) d \xi+\frac{F_{2}}{\varphi(\lambda)} f(x, \lambda) .
$$

Theorem 1 is proved.

Lemma 1: Let the function $F_{1}(x)$ is twice continuously differentiable and finite at infinity. Then as $|\lambda| \rightarrow \infty, \operatorname{Im} \lambda \geq 0$ the following is valid:

$$
\begin{aligned}
\langle G, F\rangle & =\int_{0}^{\infty} G(x, \xi ; \lambda) F_{1}(\xi) \rho(\xi) d \xi+\frac{F_{2}}{\varphi(\lambda)} f(x, \lambda) \\
& =-\frac{F_{1}(x)}{\lambda^{2}}+O\left(\frac{1}{\lambda^{2}}\right) .
\end{aligned}
$$

Proof :Using Theorem 1 and integrating by parts, we write

$$
\begin{gathered}
\int_{0}^{\infty} G(x, \xi ; \lambda) F_{1}(\xi) \rho(\xi) d \xi=-\int_{x}^{\infty} \frac{\omega(x, \lambda) f(\xi, \lambda)}{\varphi(\lambda)} F_{1}(\xi) \rho(\xi) d \xi \\
-\int_{0}^{x} \frac{\omega(\xi, \lambda) f(x, \lambda)}{\varphi(\lambda)} F_{1}(\xi) \rho(\xi) d \xi \\
=\frac{1}{\lambda^{2}} \int_{x}^{\infty} \frac{\omega(x, \lambda)}{\varphi(\lambda)}\left\{f^{\prime \prime}(\xi, \lambda)-q(\xi) f(\xi, \lambda)\right\} F_{1}(\xi) d \xi \\
+\frac{1}{\lambda^{2}} \int_{0}^{x} \frac{f(x, \lambda)}{\varphi(\lambda)}\left\{\omega^{\prime \prime}(\xi, \lambda)-q(\xi) \omega(\xi, \lambda)\right\} F_{1}(\xi) d \xi \\
=\frac{W\{\omega(x, \lambda), f(x, \lambda)\}}{\lambda^{2} \varphi(\lambda)} F_{1}(x)+\frac{1}{\lambda^{2}} F_{1}^{\prime}(0) f(x, \lambda) \\
-\frac{1}{\lambda^{2} \varphi(\lambda)} \int_{0}^{\infty} G(x, \xi ; \lambda) F_{1}(\xi) d \xi . \\
F_{1}(\xi)=-F_{1}^{\prime \prime}(\xi)+q(\xi) F_{1}(\xi) .
\end{gathered}
$$

Therefore,

$$
\int_{0}^{\infty} G(x, \xi ; \lambda) F_{1}(\xi) \rho(\xi) d \xi+\frac{F_{2}}{\varphi(\lambda)} f(x, \lambda)=
$$




$$
-\frac{F_{1}(x)}{\lambda^{2}}+\frac{1}{\lambda^{2}}\left[\frac{F_{1}^{\prime}(0)}{\varphi(\lambda)} f(x, \lambda)-\int_{0}^{\infty} G(x, \xi ; \lambda) F_{1}(\xi) d \xi\right] .
$$

Lemma 1 is proved.

\section{The Expansion Formula}

In this section, we obtain the expansion formula by applying the method of Titchmarsh.

$$
F(x, \lambda)= \begin{cases}\left(G_{x, \lambda}, F\right), & \operatorname{Im} \lambda \geq 0 \\ \left(G_{x, \bar{\lambda}}, F\right), & \operatorname{Im} \lambda \leq 0 .\end{cases}
$$

Let us $\Gamma_{R}$ denote the circle of radius $R$ and center is zero which boundary contour is positive oriented. Let us $\Gamma_{R, \varepsilon}$, denote boundary contour positive oriented in plane

$D=\{z|| z|\leq R,| \operatorname{Im} z \mid \geq \varepsilon\}$.

$\Gamma_{R, \varepsilon}^{\prime}$ denotes boundary contour negative oriented in the plane $D=\{z|| z|\leq R,| \operatorname{Im} z \mid \leq \varepsilon\}$. Then, we can use the properties of the integration as follows:

$$
\int_{\Gamma_{R, \varepsilon}}=\int_{\Gamma_{R}}+\int_{\Gamma_{R, \varepsilon}^{\prime}}
$$

Now, multiplying both sides of equality (16) by $\frac{1}{2 \pi i} \lambda$ and integrating over $\lambda$ the contour $\Gamma_{R, \varepsilon}$, we obtain

$\frac{1}{2 \pi i} \int_{\Gamma_{R, \varepsilon}} \lambda F(x, \lambda) d \lambda=-\frac{1}{2 \pi i} \int_{\Gamma_{R, \varepsilon}} \frac{F_{1}(x)}{\lambda} d \lambda+\frac{1}{2 \pi i} \int_{\Gamma_{R, \varepsilon}} O\left(\frac{1}{\lambda}\right) d \lambda$.

According to the equation (17), we obtain

$\frac{1}{2 \pi i} \int_{\Gamma_{R, \varepsilon}} \lambda F(x, \lambda) d \lambda=\frac{1}{2 \pi i} \int_{\Gamma_{R}} \lambda F(x, \lambda) d \lambda+\frac{1}{2 \pi i} \int_{\Gamma_{R, \varepsilon}^{\prime}} \lambda F(x, \lambda) d \lambda$.

Now let us calculate the integral on the right hand side of (18),

$\frac{1}{2 \pi i} \int_{\Gamma_{R}} \lambda F(x, \lambda) d \lambda=-\frac{1}{2 \pi i} \int_{\Gamma_{R}} \frac{F_{1}(x)}{\lambda} d \lambda+\frac{1}{2 \pi i} \int_{\Gamma_{R}} O\left(\frac{1}{\lambda}\right) d \lambda \underset{R \rightarrow \infty}{\rightarrow}-F_{1}(x)$.

Taking (19) into consideration, we get

$\lim _{\substack{R \rightarrow \infty \\ \varepsilon \rightarrow 0}} \frac{1}{2 \pi i} \int_{\Gamma_{R, \varepsilon}} \lambda F(x, \lambda) d \lambda=-F_{1}(x)+\frac{1}{2 \pi i} \int_{-\infty}^{\infty} \lambda[F(x, \lambda+i 0)-F(x, \lambda-i 0)] d \lambda$.

On the other hand, using the residue calculus, we obtain

$$
\frac{1}{2 \pi i} \int_{\Gamma_{R, \varepsilon}} \lambda F(x, \lambda) d \lambda=\sum_{j=1}^{n} \operatorname{Re}_{\lambda=i \lambda j}[\lambda F(x, \lambda)]+\sum_{j=1}^{n} \operatorname{Re}_{\lambda=-i \lambda j}[\lambda F(x, \lambda)] .
$$

From (21) and (22), we obtain

$$
\begin{aligned}
F_{1}(x) & =-\sum_{j=1}^{n} \operatorname{Re}_{\lambda=i \lambda_{j}}[\lambda F(x, \lambda)]-\sum_{j=1}^{n} \operatorname{Re} s_{\lambda=-i \lambda j}[\lambda F(x, \lambda)] \\
& +\frac{1}{2 \pi i} \int_{-\infty}^{\infty} \lambda[F(x, \lambda+i 0)-F(x, \lambda-i 0)] d \lambda .
\end{aligned}
$$

Let $\psi(x, \lambda)$ be the solution of (5) satisfying the initial conditions

$$
\psi(0, \lambda)=0, \psi^{\prime}(0, \lambda)=1
$$

It is clear that $W[\omega(x, \lambda), \psi(x, \lambda)]=1$.

Then,for $\operatorname{Im} \lambda>0, f(x, \lambda)$ is written as the linear combination with the solutions $\omega(x, \lambda)$ and $\psi(x, \lambda)$, i.e.

$$
f(x, \lambda)=c_{1} \omega(x, \lambda)+c_{2} \psi(x, \lambda),
$$

where

$$
c_{1}=f(0, \lambda), \quad c_{2}=-\varphi(\lambda)
$$

Hence,

$$
f(x, \lambda)=f(0, \lambda) \omega(x, \lambda)-\varphi(\lambda) \psi(x, \lambda) .
$$

Consequently, it is clear that

$G(x, \xi ; \lambda)=-\frac{1}{\varphi(\lambda)} \begin{cases}\omega(x, \lambda)[f(0, \lambda) \omega(\xi, \lambda)-\varphi(\lambda) \psi(\xi, \lambda)], & x \leq \xi<\infty, \\ {[f(0, \lambda) \omega(x, \lambda)-\varphi(\lambda) \psi(x, \lambda)] \omega(\xi, \lambda),} & 0 \leq \xi \leq x .\end{cases}$

$$
\begin{gathered}
=-\frac{f(0, \lambda)}{\varphi(\lambda)} \omega(x, \lambda) \omega(\xi, \lambda)- \begin{cases}\omega(x, \lambda) \psi(\xi, \lambda), & x \leq \xi<\infty, \\
\psi(x, \lambda) \omega(\xi, \lambda), & 0 \leq \xi \leq x .\end{cases} \\
F(x, \lambda)=-\frac{1}{\varphi(\lambda)} f(0, \lambda) \omega(x, \lambda) \int_{0}^{\infty} \omega(\xi, \lambda) F_{1}(\xi) \rho(\xi) d \xi \\
-\psi(x, \lambda) \int_{0}^{x} \omega(\xi, \lambda) F_{1}(\xi) \rho(\xi) d \xi-\omega(x, \lambda) \int_{x}^{\infty} \psi(\xi, \lambda) F_{1}(\xi) \rho(\xi) d \xi \\
+\frac{F_{2}}{\varphi(\lambda)} f(0, \lambda) \omega(x, \lambda)-F_{2} \psi(x, \lambda) .
\end{gathered}
$$

It follows that

$$
\begin{gathered}
\operatorname{Re} s_{\lambda=i \lambda_{j}}[\lambda F(x, \lambda)]=-\frac{i \lambda_{j}}{\dot{\varphi}\left(i \lambda_{j}\right)} f\left(0, i \lambda_{j}\right) \omega\left(x, i \lambda_{j}\right) \int_{0}^{\infty} \omega\left(\xi, i \lambda_{j}\right) F_{1}(\xi) d \xi \\
+\frac{i \lambda_{j} F_{2}}{\dot{\varphi}\left(i \lambda_{j}\right)} f\left(0, i \lambda_{j}\right) \omega\left(x, i \lambda_{j}\right), j=1,2, \ldots, n .
\end{gathered}
$$

Using properties of $f(x, \lambda)$ and $\omega(x, \lambda)$, we get

$$
f\left(x, \lambda_{j}\right)=f\left(0, \lambda_{j}\right) \omega\left(x, \lambda_{j}\right), j=1,2, \ldots, n .
$$

From (9) and (25) we obtain 


$$
\begin{aligned}
& \operatorname{Re}_{\lambda=i \lambda_{j}}[\lambda F(x, \lambda)]+\operatorname{Re}_{\lambda=-i \lambda_{j}}[\lambda F(x, \lambda)] . \\
& =-m_{j}^{2} f\left(x, i \lambda_{j}\right) \int_{0}^{\infty} f\left(\xi, i \lambda_{j}\right) F_{1}(\xi) d \xi+m_{j}^{2} F_{2} f\left(0, i \lambda_{j}\right) f\left(x, i \lambda_{j}\right) . \\
& =-m_{j}\left(F, U_{j}(x)\right) f\left(x, i \lambda_{j}\right),
\end{aligned}
$$

where

$$
U_{j}(x)=m_{j}\left(\begin{array}{c}
f\left(x, i \lambda_{j}\right) \\
f\left(0, i \lambda_{j}\right)
\end{array}\right), F(x)=\left(\begin{array}{c}
F_{1}(x) \\
F_{2}
\end{array}\right), j=1,2, \ldots, n .
$$

Now, let us calculate the integral

$$
\frac{1}{2 \pi i} \int_{-\infty}^{\infty} \lambda[F(x, \lambda+i 0)-F(x, \lambda-i 0)] d \lambda .
$$

From formula (24) and equality $F(x, \lambda-i 0)=\overline{F(x, \lambda+i 0)}$, we get

$$
\begin{aligned}
& F(x, \lambda+i 0)-F(x, \lambda-i 0)= \\
& {\left[\frac{1}{\overline{\varphi(\lambda)}} \overline{f(0, \lambda)}-\frac{1}{\varphi(\lambda)} f(0, \lambda)\right] \omega(x, \lambda) \times \int_{0}^{\infty} \omega(\xi, \lambda) F_{1}(\xi) \rho(\xi) d \xi} \\
& =\frac{2 i \lambda}{|\varphi(\lambda)|^{2}} \omega(x, \lambda) \int_{0}^{\infty} \omega(\xi, \lambda) F_{1}(\xi) \rho(\xi) d \xi-\frac{2 i \lambda F_{2}}{|\varphi(\lambda)|^{2}} \omega(x, \lambda) .
\end{aligned}
$$

It follows that

$$
\begin{gathered}
\frac{1}{2 \pi i} \int_{-\infty}^{\infty} \lambda[F(x, \lambda+i 0)-F(x, \lambda-i 0)] d \lambda= \\
\frac{2}{\pi} \int_{0}^{\infty} \frac{\lambda^{2}}{|\varphi(\lambda)|^{2}} \omega(x, \lambda) \int_{0}^{\infty} \omega(\xi, \lambda) F_{1}(\xi) \rho(\xi) d \xi-\frac{2 F_{2}}{\pi} \int_{0}^{\infty} \frac{\lambda^{2} \omega(x, \lambda)}{|\varphi(\lambda)|^{2}} d \lambda .
\end{gathered}
$$

Taking (26), (27) into (23), we obtain the expansion formula according to the eigenfunctions:

$$
\begin{gathered}
F_{1}(x)=\sum_{j=1}^{n} m_{j}\left(F, U_{j}(x)\right) f\left(x, i \lambda_{j}\right) \\
+\frac{2}{\pi} \int_{0}^{\infty} \frac{\lambda^{2} d \lambda}{|\varphi(\lambda)|^{2}} \omega(x, \lambda) \int_{0}^{\infty} \omega(\xi, \lambda) F_{1}(\xi) \rho(\xi) d \xi d \lambda \\
-\frac{2 F_{2}}{\pi} \int_{0}^{\infty} \frac{\lambda^{2} \omega(x, \lambda)}{|\varphi(\lambda)|^{2}} d \lambda . \\
F_{2}=\beta_{1} F_{1}(0)=\sum_{j=1}^{n} m_{j} \beta_{1}\left(F, U_{j}(0)\right) f\left(0, i \lambda_{j}\right) \\
+\frac{2 \beta_{1}}{\pi} \int_{0}^{\infty} \frac{\lambda^{2} d \lambda}{|\varphi(\lambda)|^{2}} \int_{0}^{\infty} \omega(\xi, \lambda) F_{1}(\xi) \rho(\xi) d \xi d \lambda \\
-\frac{2 \beta_{1} F_{2}}{\pi} \int_{0}^{\infty} \frac{\lambda^{2}}{|\varphi(\lambda)|^{2}} d \lambda .
\end{gathered}
$$

\section{References}

[1] D. S. Cohen, "An Integral Transform Associated with Boundary Conditions Containing an Eigenvalue Parameter," SIAM Journal on Applied Mathematics, Vol. 14, No. 5 (Sep., 1966), 1164-1175

[2] C. T. Fulton, "Two-Point Boundary Value Problems with Eigenvalue Parameter Contained in the Boundary Conditions," In. Proc. Roy. Soc. Edin., 77 A, (1977), 293-308.

[3] C. T. Fulton, "Singuler Eigenvalue Problems with Eigenvalue Parameter Contained in the Boundary Conditions," In. Proc. Roy. Soc. Edin., Section A, Vol. 87, No. 1-2, pp. 1-34, 1980/1

[4] Levitan B. M., Sargsjan I. S., Introduction to Spectral Theory, American Mathematical Society, 1975. 
[5] E. C. Titchmarsh, Eigenfunction Expansions, Oxford, (1962)

[6] Naimark M. A., Linear Differantial Operators, Frederick Ungar Publishing, 1967.

[7] V. A. Marchenko, Sturm-Liouville Operators and Applications, AMS Chelsea Publishing, 2011

[8] Zettl A., Sturm-Liouville Theory, Mathematical Surveys and Monographs 121, American Math. Soc., Providince, RI, 2005 .

[9] J. Wedmann, Spectral Theory of Ordinary Differential Operators, Lecture Notes in Math. 1258, Springer-Verlag, Berlin, 1987.

[10] Kh. R. Mamedov and N. Palamut, "On a Direct Problem of Scattering Theory For A Class Of Sturm Liouville Operator With Discontinous Coefficient," Proccedings of the Jangjean Mathematical Society, vol. 12, no.2, pp. 243-251, 2009.
[11] Kh. R. Mamedov, "Uniqueness of the Solution of the Inverse Problem of Scattering Theory for Sturm-Liouville Operator with a Spectral Parameter in the Boundary Condition, In: Math. Notes, V. 74 No:1, (2003), 136-140.

[12] Kh. R. Mamedov, "Uniqueness of the Solution of the Inverse Problem of Scattering Theory for Sturm-Liouville Operator with Discontinous Coefficient," In: Proceedings of IMM of NAS Azerbaijan, 24, (2006), 263-272.

[13] Kh. R. Mamedov, "On An Inverse Scattering Problem For A Discontinuous Sturm-Liouville Equation With A Spectral Parameter in the Boundary Condition," Boundary Value Problems Volume 2010, article d 171967, 17 pages, doi $10.1155 / 2010 / 171967$

[14] M. Guseinov, R.T. Pashaev, "On an Inverse Problem for a Second Order Differential Equation," In: Usp. Math. Nauk, V 57 no: 3, (2002), 597-598J. 